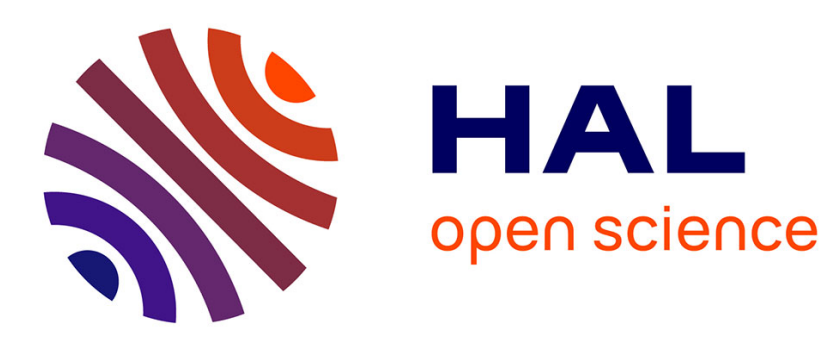

\title{
A stochastic data-based traffic model applied to vehicles energy consumption estimation
}

\author{
Arthur Le Rhun, Frédéric Bonnans, Giovanni de Nunzio, Thomas Leroy, \\ Pierre Martinon
}

\section{- To cite this version:}

Arthur Le Rhun, Frédéric Bonnans, Giovanni de Nunzio, Thomas Leroy, Pierre Martinon. A stochastic data-based traffic model applied to vehicles energy consumption estimation. IEEE Transactions on Intelligent Transportation Systems, In press, 10.1109/TITS.2019.2923292 . hal-01774621v3

\section{HAL Id: hal-01774621 \\ https://hal.inria.fr/hal-01774621v3}

Submitted on 4 Sep 2019

HAL is a multi-disciplinary open access archive for the deposit and dissemination of scientific research documents, whether they are published or not. The documents may come from teaching and research institutions in France or abroad, or from public or private research centers.
L'archive ouverte pluridisciplinaire HAL, est destinée au dépôt et à la diffusion de documents scientifiques de niveau recherche, publiés ou non, émanant des établissements d'enseignement et de recherche français ou étrangers, des laboratoires publics ou privés. 


\title{
A stochastic data-based traffic model applied to vehicles energy consumption estimation
}

\author{
Arthur Le Rhun, Frédéric Bonnans, Giovanni De Nunzio, Thomas Leroy, Pierre Martinon
}

\begin{abstract}
A new approach to estimate traffic energy consumption via traffic data aggregation in (speed,acceleration) probability distributions is proposed. The aggregation is done on each segment composing the road network. In order to reduce data occupancy, clustering techniques are used to obtain meaningful classes of traffic conditions. Different times of the day with similar speed patterns and traffic behavior are thus grouped together in a single cluster. Different energy consumption models based on the aggregated data are proposed to estimate the energy consumption of the vehicles in the road network. For validation purposes, a microscopic traffic simulator is used to generate the data and compare the estimated energy consumption to the measured one. A thorough sensitivity analysis with respect to the parameters of the proposed method (i.e. number of clusters, size of the distributions support, etc.) is also conducted in simulation. Finally, a real-life scenario using floating car data is analyzed to evaluate the applicability and the robustness of the proposed method.
\end{abstract} tion

Index Terms-Traffic modeling, Clustering, Energy Consump-

\section{INTRODUCTION}

I N 2015, according to data from the European Environment Agency, road transportation contributed to $21 \%$ of total EU28 greenhouse gas emissions. In order to meet the long-term emissions reduction target, emissions from transportation need to fall by more than two thirds by 2050 [1]. These emissions are essentially a function of the vehicle propulsion technology and the driving style [2].

Estimating energy consumption of the vehicles is a great challenge in the objective of improving global transportation efficiency, since this information is used in energy management, eco-routing, eco-driving, traffic management, ... Traffic congestion has a major impact on the driving behavior, and thus plays a key role in the level of fuel consumption [3].

Therefore, accurate predictions of vehicles energy consumption must take traffic conditions into account. To perform this objective, faithful modeling of traffic behavior is of primary importance. Energy-oriented modeling approaches can be divided in two main categories.

On the one hand, several mathematical traffic models are available nowadays, see for instance [4]. Such models typically

A. Le Rhun, IFP Energies nouvelles, 1 avenue de Bois-Préau, 92852 RueilMalmaison, France, arthur.le-rhuneinria.fr

G. De Nunzio, IFP Energies nouvelles, Rond-point de l'échangeur de Solaize, BP 3, 69360 Solaize, France, giovanni. de-nunziod ifpen. fr

T. Leroy, IFP Energies nouvelles, 1 avenue de Bois-Préau, 92852 RueilMalmaison, France, thomas. leroyeifpen. fr

F. Bonnans, Inria Saclay and CMAP Ecole Polytechnique, route de Saclay, 91128 Palaiseau, France, frederic.bonnanseinria.fr

P. Martinon, Inria Saclay and CMAP Ecole Polytechnique, route de Saclay, 91128 Palaiseau, France, pierre.martinon@inria.fr depict the reality either from a macroscopic point of view, based on the road vehicular density [5], or from a microscopic perspective, based on the description of the instantaneous behavior of each vehicle [6]. Both approaches have limitations in providing an accurate energy consumption estimation. Macroscopic models typically provide average traffic speeds to compute energy consumption [7], thus neglecting the impact of speed fluctuations due to congestion. Higher precision of the energy consumption estimation could only be obtained at the expense of a denser discretization of the road network, therefore compromising scalability. Microscopic models could achieve precise energy consumption estimation, but they require a significant calibration and validation effort. Also, the computational burden and the amount of collected data grows rapidly with the size of the network, therefore these models are more suitable for off-line use.

On the other hand, data-based models rely on collected traffic information to estimate traffic behavior and energy consumption. Instantaneous models are able to precisely estimate energy consumption by using large amounts of data, generally the measured driving profile of each vehicle. To tackle this drawback, aggregated models use the average value of the measured speed profiles to compute energy consumption, but they suffer from the same accuracy problems previously discussed for the macroscopic traffic models. Furthermore, the data sparsity and availability is an issue [8]. Other approaches try to solve the problem of the data sparsity by simply classifying road segments by category (e.g. urban, arterial, freeway, etc.), in order to associate each category with a typical energy use. This type of models may lead to inaccuracy in energy consumption estimation, as road segments belonging to the same category may show very different traffic patterns [9].

In this work, a new way to represent traffic behavior on large road networks is proposed. The objective of this model is to accurately depict the effect of traffic conditions on the vehicles energy consumption in each road segment. The key idea is to use a statistical approach based on vehicle speed and acceleration data, measured from real vehicles. In particular, the entire observation time during which speed and acceleration data are collected is subdivided into time-frames. During each time-frame a (speed,acceleration) distribution is generated, and such a distribution is then used as an input for an energy consumption model to estimate the traffic energy consumption on the analyzed road segment during the specific time-frame. Therefore, each road segment is defined by its own collection of (speed,acceleration) distributions. 
The proposed model also includes the possibility of reducing the dimension of the traffic data and increasing scalability, by applying clustering techniques to the probability distributions of each road segment. For instance, the different distributions representing traffic in one road segment over different hours of the day may be aggregated in clusters modeling only significant traffic conditions (e.g. peak, offpeak, etc.).

The paper is organized as follows. The clustering technique and the proposed energy consumption model are presented in Section [I] The traffic data collection and the model validation procedure are discussed in Section III Using traffic data from the simulator SUMO, Section IV] illustrates the method first on a single road segment, then for a larger set of segments. Finally, section $\mathrm{V}$ presents an application of the method to actual traffic data, as well as a comparison to a more basic approach, using only mean speeds.

\section{Proposed Method}

\section{A. Road segments}

In the following, the road network is assumed to be subdivided into a collection of segments. A segment is a portion of road with homogeneous topographic characteristics. Segments are typically delimited by network elements such as traffic lights, crossroads, or roundabouts. With a traffic simulator such as SUMO we can retrieve the segments from the simulator model, see IV-A When working on actual traffic data, segments can be obtained from Geographic Information Systems (GIS) data (e.g. OpenStreetMap, HERE Maps, etc.). Segments length typically range between a few meters and a few hundreds meters. In the following we denote $S$ the set of road segments, of size $N_{S}$.

\section{B. Probability distribution for (speed,acceleration)}

The consumption of an engine depends on its operating point, which can be determined by the speed and acceleration of the vehicle. The key point in our method is to assume that an accurate (with respect to energy consumption) description of the traffic can be derived from the probability distributions of measured speeds $v$ and accelerations $a$ for each road segment. Note that these distributions do not retain the temporality of the speed profiles.

We set $N_{T}$ the number of time-frames for each segmen $1{ }^{1}$ We denote the family of time-frames $\left(t_{i, s}\right)_{\left(1 \ldots N_{t}\right) \times\left(1 \ldots N_{S}\right)}$,

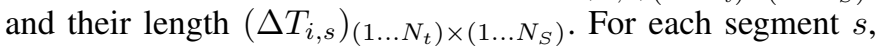
we record the (speed,acceleration) of all the vehicles passing through the segment during all the time-frames $t_{i, s}$.

In the following, we work with discrete distributions in the $(v, a)$ space. We denote $\mathbb{V}$ and $\mathbb{A}$ the sets of feasible speed and acceleration. To simplify, these sets are taken identical for all segments and time-frames, thus all (speed,acceleration) discrete probability distributions have the same support in $\mathbb{V} \times \mathbb{A}$. We denote $N_{\mathbb{V}}$ and $N_{\mathbb{A}}$ the discretization size of $\mathbb{V}$ and $\mathbb{A}$. Recalling that $N_{S}$ and $N_{T}$ are the number of road

\footnotetext{
${ }^{1}$ For the sake of simplicity, we assume the same number of time-frames for all the segments
}

segments and time-frames, we obtain a total of $N_{S} N_{T}$ discrete distributions of support size $N_{\mathbb{V}} N_{\mathbb{A}}$. Fig 1 shows an example of such a distribution.

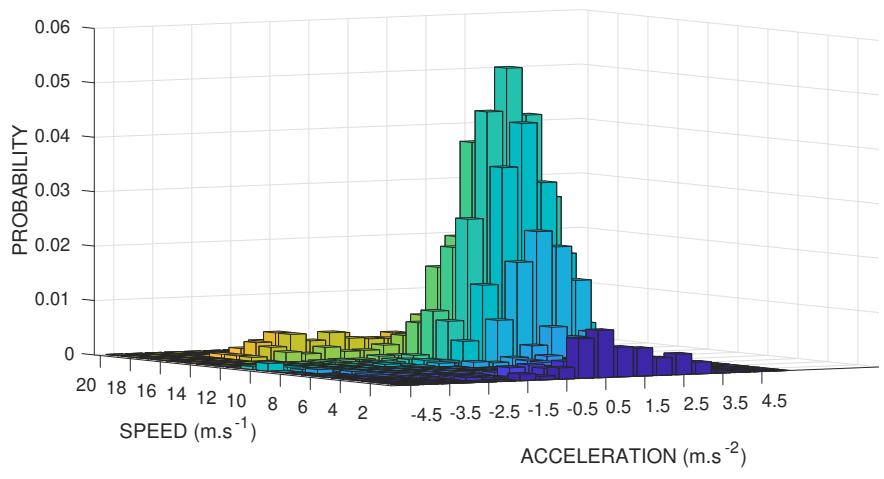

Fig. 1: Example of (speed,acceleration) distribution.

\section{K-means clustering with strong patterns}

For each segment $s$ and time-frame $t_{i, s}$ we have a probability distribution $\mu_{i, s}$. When considering large road networks with several thousands of segments, the data size grows rapidly to several gigabytes. A natural idea is to reduce the information through clustering techniques applied to these distributions. Then, for each cluster $j$, we can take its barycenter $\overline{\mu_{j}}$ as representative of the traffic conditions for all the segment/time-frame pairs $\left(s, t_{i, s}\right)$ that belong to this cluster. This way, we only have to store the distributions corresponding to the barycenters of the clusters.

Since the elements to be clustered are probability distributions, we use the 1-Wasserstein distance ( [10], [11]). This distance based on optimal transport theory tends to preserve the geometrical aspects (shapes) of the distributions. We recall the definition of the Wasserstein distance:

$$
W_{1}(\mu, \nu)=\min _{\pi \in \Pi(\mu, \nu)} d \cdot \pi
$$

where $\Pi(\mu, \nu)$ is the set of transportation plans from $\mu$ to $\nu$, i.e. the set of nonnegative matrices $\pi$ of size $\left(\left|N_{\mathbb{V}}\right| \times\left|N_{\mathbb{A}}\right|\right)^{2}$ with marginals $\pi^{\top} \mathbf{1}=\mu$ and $\pi \mathbf{1}=\nu$. As speed and acceleration have comparable magnitude orders when expressed in SI units, we simply define the displacement cost $d$ by the Euclidean norm between the origin and destination points.

The notion of barycenter for distributions has been extended to the Wasserstein distance in [12], where the barycenter is defined as the distribution that minimizes the sum of the squared Wasserstein distances to all distributions of the set. For the practical aspects we follow [13] in which the barycenter minimizes the sum of $W_{\epsilon}$ distances, with $W_{\epsilon}$ an entropic regularization of the Wasserstein distance, computed by a Sinkhorn-type algorithm.

We use the K-means algorithm [14] to compute the clusters, and note $k$ the (fixed) number of clusters. To reduce the 
sensitivity of the K-means algorithm to the initial guess, we use the strong patterns method [15]. Strong patterns are subsets whose elements always end up in the same cluster regardless of the K-means starting point. In practice, we run a first batch of K-means with random initializations to identify the strong patterns, and then perform a final K-means which is initialized by taking one element in each of the $k$ largest strong patterns. The principle of the K-means is recalled in Algorithm 1

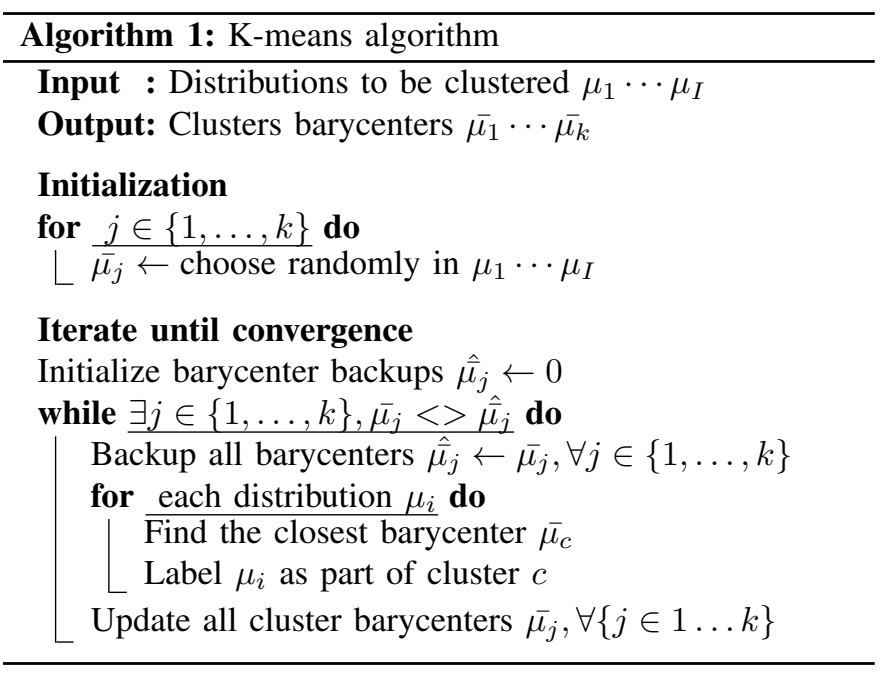

\section{Computing energy consumption}

The energy consumption computed is the energy at the wheel, neglecting the losses due to the powertrain. The instantaneous power at the wheel is denoted as a general function $P(v, a)$, which can be for instance of the form presented in (5). The ultimate objective of the proposed method is to estimate energy consumption by using only the information extracted from the $(v, a)$ probability distributions $\mu$. More accurately, we seek to obtain the energy consumption from the barycenter $\bar{\mu}$ of the cluster containing $\mu$. In the following we introduce two methods to compute the consumption of a generic vehicle passing through a segment. These will be referred to as "Average Consumption Method" and "Memoryless Sampling Method".

1) Average Consumption method: The first idea is to use the average power $\bar{P}$ (in the probabilistic sense)

$$
\bar{P}\left(\bar{\mu}_{t}\right)=\sum_{\mathbb{V} \times \mathbb{A}} \bar{\mu}_{t}(v, a) P(v, a)
$$

where $\bar{\mu}_{t}$ is the barycenter of the cluster containing the current segment at time $t$. The barycenter may indeed change if $t$ crosses different time-frames while the vehicle is on the segment. This average power is integrated over the time interval $\left[t_{i}, t_{f}\right]$ spent by the vehicle on the segment, thus

$$
C_{A v g}=\int_{t_{i}}^{t_{f}} \bar{P}\left(\overline{\mu_{t}}\right) d t
$$

Note that knowledge of the time interval is required in this method, in addition to the (speed,acceleration) distributions.
Indeed, using here the average time would give identical consumption for every vehicle. Therefore, we need some more statistically significant time information in order to capture the deviation of the consumption distribution. A resulting drawback of this method is that a faster vehicle has a shorter travel time and thus a lower energy consumption, which may seem unrealistic.

2) Memoryless Sampling method: In the second method the energy consumption is still obtained by integrating the instantaneous power, but we do not use the average power. Instead, we implement the idea that the vehicle must follow the traffic at every time, in a statistical sense. More precisely, its speed and acceleration should follow the probability distribution of the barycenter $\bar{\mu}$ of the cluster for the current pair $(s, t)$. Another difference is that the integration is performed over the segment length $L_{s}$ instead of travel time.

So the Memoryless Sampling method generates a sequence of $\left(v_{n}, a_{n}\right)$, independent samples according to the probability distribution $\bar{\mu}$. Setting a time step $\delta t$, we use this sequence to integrate both the traveled distance and the instantaneous power. We assume $\delta t=1 s$, in order to have the same order of magnitude as the reaction time of a driver. We stop the generation of $\left(v_{n}, a_{n}\right)$ when the vehicle reaches the end of the segment 2 Since the distance will be covered in a finite time, we obtain the finite set of samples $\left(v_{n}, a_{n}\right)_{n=1, \ldots, n_{f}}$ and the consumption writes as

$$
C_{M S M}=\sum_{n=1}^{n_{f}} P\left(v_{n}, a_{n}\right) \delta t
$$

\section{VALIDATION APPROACH}

\section{A. Power and Reference energy consumption}

In order to assess the accuracy of our two methods (Average and Memoryless Sampling), both based on the statistical representation of traffic, we introduce a "reference" energy consumption for the sake of comparison. First we choose a simple equation for the instantaneous power $P(v, a)$, neglecting all road slope effects:

$$
P(v, a)=\left(m a+a_{2} v^{2}+a_{1} v+a_{0}\right) v
$$

where $m$ is the vehicle mass and $a_{0}, a_{1}, a_{2}$ define a vehicledependent polynomial approximation of the road-load force. We use the numerical values $m=1400 \mathrm{~kg}, a_{0}=185.4, a_{1}=$ $0, a_{2}=0.3$, corresponding to a passenger vehicle [16]. We would like to point out that energy consumption models are a wide topic, and we refer interested readers for instance to [17]. In our case we essentially want to compare the consumptions from raw traffic data and statistically processed data, so the key point is to use the same power expression for all consumption formulas. In all the following, these energy consumptions $C$ are computed by integrating ${ }^{3}$ the instantaneous power $P$ along the (speed,acceleration) profiles, without taking into account any regenerative braking, namely

$$
C=\int_{t_{i}}^{t_{f}} \max (0, P(\mathbf{v}(t), \mathbf{a}(t))) \mathrm{dt}
$$

${ }^{2}$ which happens with probability 1 since vehicles never stop indefinitely.

${ }^{3}$ in practice integration is done by the Euler scheme. 
In order to compare the different methods, we compute the distribution of the energy consumptions for a given (segment,timeframe):

- the Reference energy distribution $\mathbf{C}_{R e f}$ is obtained by plugging into (5) the recorded speed and acceleration $(\mathbf{v}(t), \mathbf{a}(t))$ of all vehicles passing through the segment during the timeframe, according to the traffic simulator.

- for the Average and Memoryless Sampling methods, we recall that the consumption distributions $\mathbf{C}_{A v g}, \mathbf{C}_{M S M}$ are obtained using the speed/acceleration probability laws $\bar{\mu}$ from the barycenters, see (3) and (4).

\section{B. Indicators}

Since our aim is to compare distributions of energy consumptions, we study several indicators.

- Mean and Standard deviation errors are classical indicators. We compute these relative errors as follows, with $\mathbf{C}_{\text {method }}$ denoting either the Average consumption or the Memoryless Sampling consumption:

$$
\begin{gathered}
\varepsilon_{\text {mean }}(s)=\frac{\overline{\mathbf{C}_{\text {method }}(s)}-\overline{\mathbf{C}_{\text {ref }}(s)}}{\overline{\mathbf{C}_{\text {ref }}(s)}} \\
\varepsilon_{\sigma}(s)=\frac{\sigma_{\mathbf{C}_{\text {method }}}(s)-\sigma_{\mathbf{C}_{\text {ref }}}(s)}{\sigma_{\mathbf{C}_{\text {ref }}(s)}}
\end{gathered}
$$

- Kullback-Leibler divergence [18], also called 'relative entropy', is a particular case of $\varphi$-divergence. KL divergence can be used to measure distances between two probability distributions $P$ and $Q$, however it is not a metric (no triangular inequality or symmetry). Another drawback is that it cannot be computed for instance when the probability of the model $q$ is 0 while the probability of the reference $p$ is not.

$$
K L(P \mid Q)=\sum_{i} p_{i} \log \left(\frac{p_{i}}{q_{i}}\right)
$$

Instead, we use the Jensen-Shannon divergence, which is a symmetrized version of KL divergence, sometimes referred to as 'total divergence to the average' [19]. Note that the square root of the JS divergence is a metric called JS distance [20], [21]. Fig 2] illustrates the JS divergence on a Gaussian with noised parameters.

$$
J S(P \mid Q)=\frac{1}{2} K L(P \mid M)+\frac{1}{2} K L(Q \mid M)
$$

with

$$
M=\frac{1}{2} P+\frac{1}{2} Q
$$

Here, we have $P=\mathbf{C}_{\text {method }}$ and $Q=\mathbf{C}_{\text {Ref }}$.

\section{RESUltS WITH SIMULATED TRAFFIC DATA}

\section{A. Traffic Data from simulation}

We illustrate our approach with data obtained from the traffic simulator SUMO [22]. The simulation runs the scenario LUST [23], which models a $24 \mathrm{~h}$ traffic in the city of Luxembourg. The time step is set to one second, i.e. $1 \mathrm{~Hz}$ sampling frequency for the variables of the vehicles. For the road segments we take the subdivision from the scenario, consisting of roughly 24000 elements. We aggregate contiguous road lanes together to obtain more data per segment, and end up

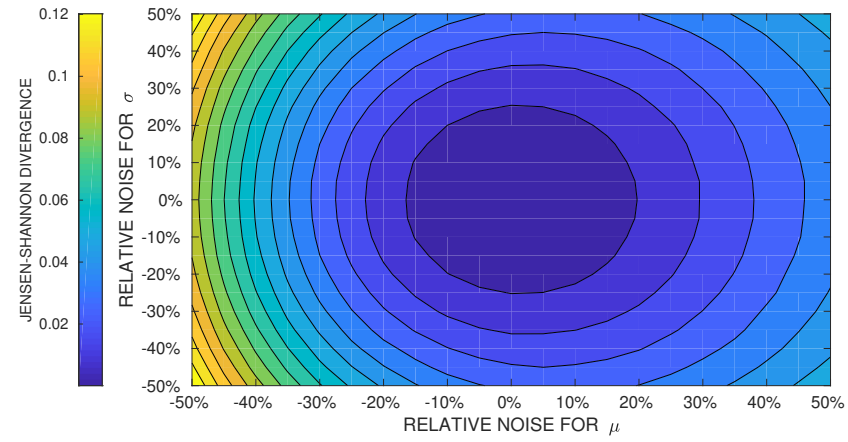

Fig. 2: Jensen-Shannon divergence for a noised Gaussian distribution with base parameters $\mu=\sigma=1$.

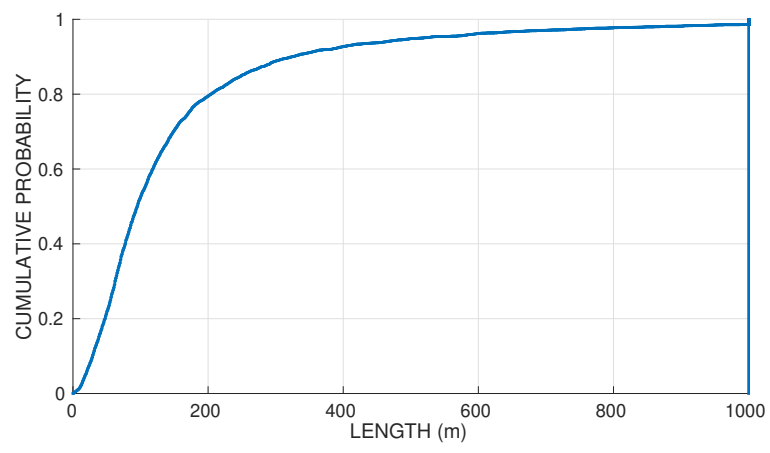

Fig. 3: An illustration of cumulative distribution of the length of road segments in city of Luxembourg as implemented in LUST for SUMO.

with 18322 segments. The length of these road segments is shown on Fig. 3

From the simulation raw data $(5.7 \mathrm{~GB})$, we extract the segment, speed, and acceleration of each vehicle in the network at all time steps. We aggregate the records for a fixed time-frame length $\Delta t_{s, i}$ (in practice we use a constant frame length $\Delta t$ for all segments). This is done both to gather sufficient data on the segments, and to decrease the number of traffic distributions for the clustering phase. For instance a time-frame of $1 \mathrm{~h}$ will give 24 distributions per segment, while 10 min gives 144 distributions per segment. In the following all codes are written in Python/NumPy and run on a standard desktop computer.

\section{B. Numerical results on one segment}

To begin with, we compare the different consumptions on a single road segment. The objects compared are therefore the consumptions distributions of all the vehicles that went through the segment during each time-frame. Results are shown as the cumulative distribution function of the consumptions for all time-frames.

We analyze in particular the influence of the (speed,acceleration) discretization, the clustering, and the choice of time-frame duration. Unless specified otherwise, the distributions are shown for a discretization $N_{V}=N_{A}=10$, a time-frame $\Delta t=10 \mathrm{~min}$, taking the full set of distributions 
without clustering.

1) Influence of the (speed,acceleration) discretization: First, Fig 4 shows the consumption distribution for both $\mathrm{t}$ Average method and Memoryless Sampling method. We t the discretizations $N_{V}=N_{A}=10,20$ and 30 and compc to the reference consumption. We see that for the Memoryle Sampling method: i) the general shape of the distribution similar to the reference and ii) finer $(v, a)$ discretizations gi distributions closer to the reference. On the other hand, 1 the Average method: i) we observe some linearization of tne consumption and ii) the effect of discretization is much less significant.
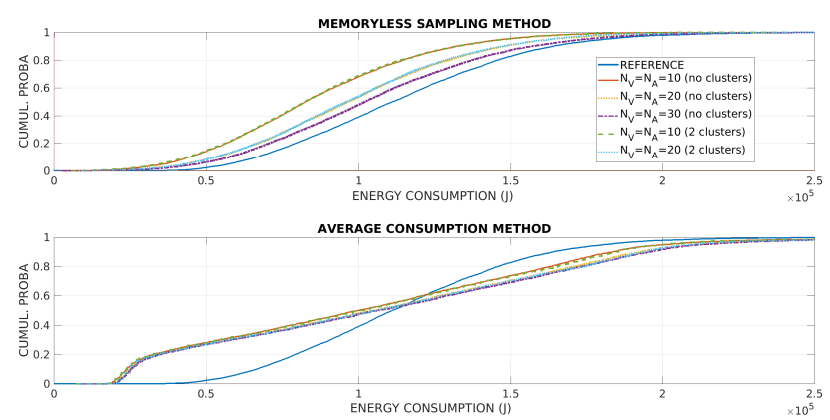

Fig. 4: Cumulated consumption distribution for one segment - effect of $(v, a)$ discretization and clustering. Reference consumption (blue line) is recomputed along each vehicle speed/acceleration profile. The two graphs show the consumptions estimated by the memoryless sample and the average consumption methods. We compare several settings for the speed/acceleration discretization, as well as the number of clusters.

2) Influence of the time-frame: Next we study the effect of the length of the time-frame $\Delta t$, which is the time interval over which we aggregate the vehicles data. Longer time-frames may cause some over-averaging and loss of specific traffic information. On the other hand, shorter time-frames may lead to insufficient vehicle data (for statistical relevance), and also increase the number of $(v, a)$ distributions to handle. Fig. 5 shows the energy consumptions obtained for time-frames of $5 \mathrm{~s}, 1 \mathrm{~min}$ and $10 \mathrm{~min}$. On this segment, for both the Average and the Memoryless Sampling methods, the influence of the time-frame duration seems rather small.

3) Influence of the clustering: To conclude this first batch of results, we examine the information loss due to the clustering stage. The consumptions obtained using only the barycenters (green and cyan curves in Fig 4) are almost identical to the ones obtained without the clustering stage. This indicates that the data reduction performed by the clustering comes with a negligible loss of information.

\section{Numerical results on 500 segments}

Now, we consider the entire road network of the city of Luxembourg, as implemented in LUST, with $\simeq 18000$ road segments. In order to study the influence of the different

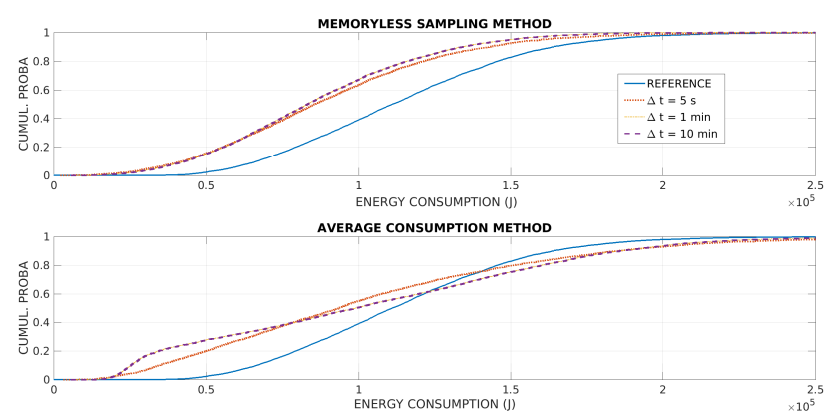

Fig. 5: Cumulated consumption distribution for one segment - effect of the time-frame duration.

parameters while keeping reasonable computational times, we pick a test set of 500 random segments, for which we perform the clustering and compute the energy consumption with the Average and Memoryless methods. For each segment, we use the indicators defined in III-B to compare the computed energy consumptions to the Reference consumption. We discuss the relevance of the models based on the distributions of these indicators on the set of segments. More precisely, we investigate the influence of the discretization of speed and acceleration, and the number of clusters. Unless specified otherwise, the simulations use a speed and acceleration discretizations of 20 steps, a time-frame of 10 minutes, with 2 clusters.

1) Influence of speed discretization: The speed discretization has a direct influence over the barycenters computed by the K-means, since it changes the support of the distribution obtained. We expect a finer discretization to give computed consumptions (Average and Memoryless Sampling methods) closer to the Reference ones. The obvious drawbacks are an increased cost of the barycenter computation and size of the distributions. We test $N_{\mathbb{V}}=5,10,20$ steps for the discretization, the speed interval being $[0,20]$ in $\mathrm{m} / \mathrm{s}$.

- Relative mean and standard deviation errors

We begin with the distribution over the 500 test segments of the mean and standard error (both relative). Fig. $6 \mathrm{a}$ and $6 \mathrm{~b}$ (upper graphs) show the errors between the Reference energy consumption and the Memoryless Sampling method. The mean and standard error both appear to be reasonably well centered around 0 . We also observe that finer discretizations of the speed clearly improve the standard error, possibly due to a better reconstruction of the travel times. On the other hand the mean error is shifted towards positive values, and is indeed smaller for 10 steps than 20.

Fig. 6a and 6b (lower graphs) compare the Average method consumptions to the Reference. Here the mean error is almost always negative, and the standard error is also negative for $80 \%$ of the segments. This strong unbalance towards the negative indicates that the Average method tends to underestimate the consumption. Increasing the speed discretization reduces the mean error, but does not really improve the standard error. A possible explanation is 

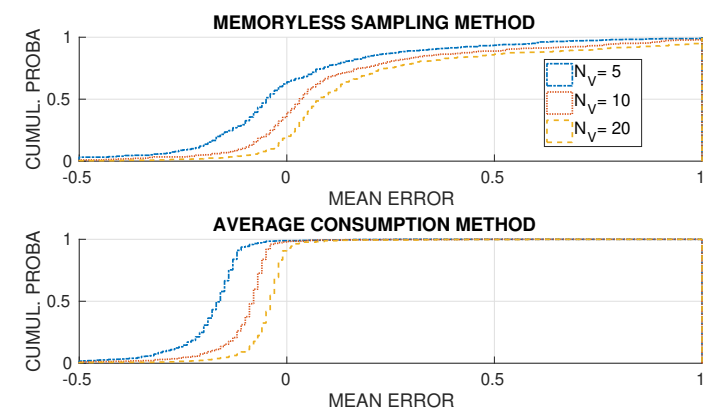

(a) Cumulated distribution of relative Mean error.
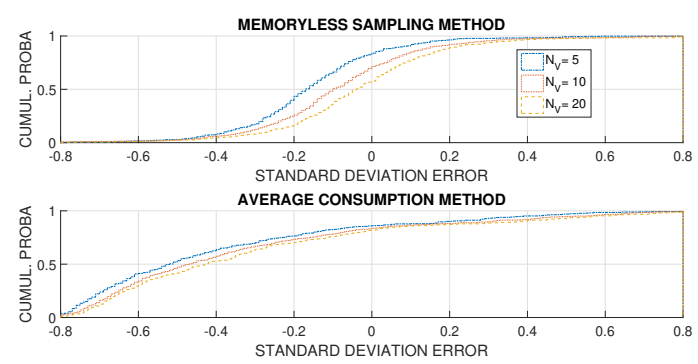

(b) Cumulated distribution of relative Standard deviation error.
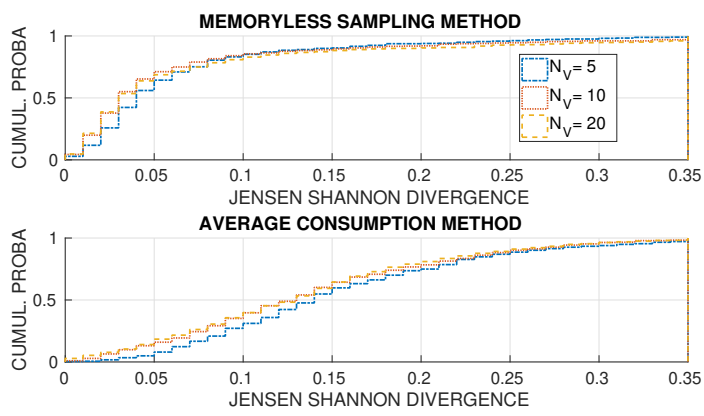

(c) Cumulated distribution of Jensen-Shannon divergence.

Fig. 6: Speed discretization.

the fact that the travel time for each vehicle is taken from the simulation, thus the speed discretization has no effect on it.

- Jensen-Shannon Divergence

Fig. 6c shows the distribution over the 500 segments of the Jensen-Shannon divergence. Upper graph is for the Memoryless Sampling versus Reference, and lower graph is for Average method versus Reference. We observe that the JS divergence is much smaller overall for the Memoryless Sampling than the Average method, with distributions more concentrated towards zero. For both methods, increasing the speed discretizations from 5 to 10 steps improves the JS divergence, while 20 steps yield very little additional gain.

2) Influence of acceleration discretization: Now we study the discretization of the acceleration. As for the speed, this parameter influences the support of the $(v, a)$ distributions, and therefore the K-means clustering. We want to know if finer discretizations of $a$ give more accurate energy consumptions for the Average and Memoryless Sampling methods. We test $N_{\mathbb{A}}=5,10,20$ steps for the discretization, the acceleration interval being $[-4.5,4.5]$ in $\mathrm{ms}^{-2}$.

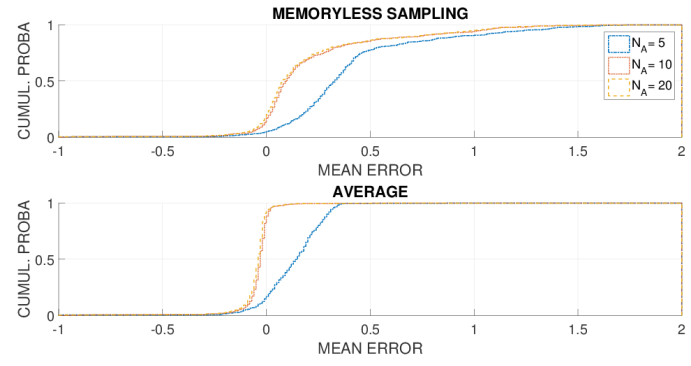

(a) Cumulated distribution of relative Mean error.
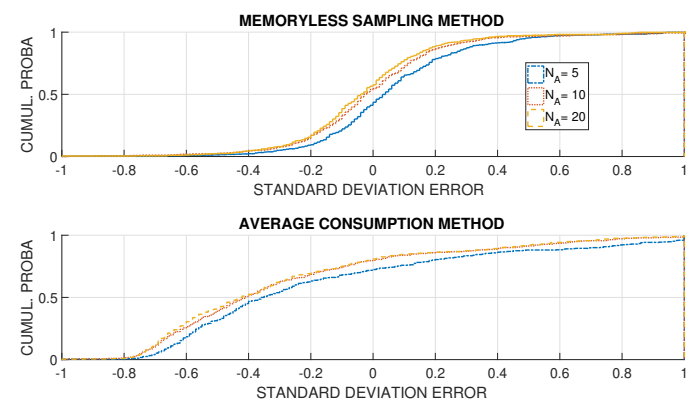

(b) Cumulated distribution of relative Standard deviation error.
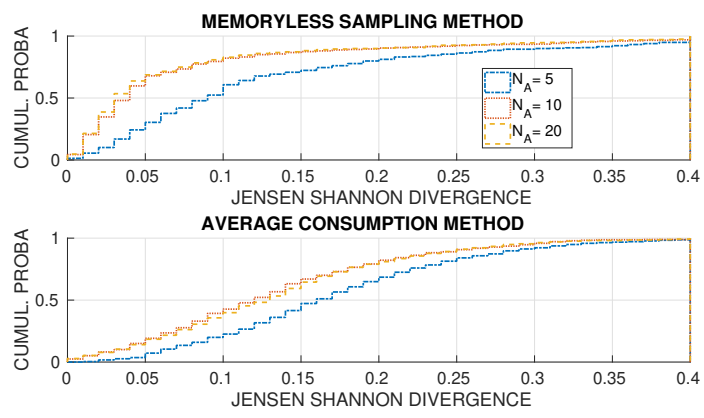

(c) Cumulated distribution of Jensen-Shannon divergence.

Fig. 7: Acceleration discretization.

- Relative mean and standard deviation errors

Fig 7a shows the mean error distribution for the two methods, with different discretizations of $a$. We observe that for both the Memoryless and Average methods, going from 5 to 10 steps gives a significant improvement, while 20 steps is similar to 10. With a sufficient discretization, the mean error is extremely good for the Average method. The Memoryless method, on the other hand, tends to slightly overestimate the consumption.

Fig $7 \mathrm{~b}$ shows the standard error distributions. As observed for the speed discretization, error for the Memoryless is well balanced while Average method has mostly negative standard errors. Finer discretizations of $a$ seem to give no improvement for the standard error. This may be due to the fact that acceleration has no influence on the travel time for either method, unlike speed which is used to reconstruct the travel times in the Memoryless method.

- Jensen-Shannon Divergence

Fig. 7c shows the distribution of the Jensen-Shannon divergence when varying the discretization of $a$. Like in the 
speed discretization study, we observe that the JS divergence is much smaller overall for the Memoryless Sampling method. Once again, for both methods increasing from 5 to 10 steps improves the indicator, while 20 steps give no additional benefit.

3) Influence of the number of clusters: Our traffic model uses clustering techniques to reduce the size of the traffic data, while retaining the useful information. One would expect some kind of trade-off, where using a larger number of clusters would keep more information at the expense of data size.

For this particular segment, simulations with $k=2,3,4$ clusters give almost identical consumption distributions. Note that these simulations were run for a time-frame $\Delta t=1 h$, due to the increased computational cost for 3 and 4 clusters. It remains to be seen whether a smaller time-frame would benefit more from a higher number of clusters. Also, the ideal number of clusters is most likely segment-dependent. Experiments with real data in Sec. V give more insight into the impact of the number of clusters.

4) Summary and performance analysis: Generally speaking, increasing the speed, acceleration, time discretization and the number of clusters will improve the accuracy of the whole approach. However, this comes at a cost, and some parameters have more impact than others. The detailed influence of the method parameters is as follows:

- speed/acceleration discretization: greater $N_{V}$ improves the Memoryless method indicators except mean error, and the Average method except std error. Greater $N_{A}$ improves all indicators except std error for Average method.

- time-frame $\Delta t$ : seems to bring little improvement to both methods with this data set.

- number of clusters $k$ : for this data set, seems to have little effect overall as well.

The drawbacks of each of these parameters are:

- finer speed/acceleration discretizations come at the cost of increased data occupancy (before and after clustering) and higher CPU time for the clustering.

- smaller time-frames increase data occupancy before clustering, clustering CPU time, and the risk of not having enough data on the segments for the distributions to be meaningful.

- a larger number of clusters leads to a higher data occupancy after clustering, and clustering CPU time.

Overall, the validation indicates that the Memoryless method has an acceptable mean error and a good std error, while the Average method has a very good mean error but a bad std error. The JS divergence is harder to interpret but is lower for the Memoryless method (with half of the distribution below 0.025) than the Average method (half of the distribution below 0.125).

At the core, we are interested in making use of the statistical information from the traffic distributions. In this respect, the Average method appears too limited, with a bad

\begin{tabular}{lll|rrr}
$N_{V \mid A}$ & $\Delta t$ & $k$ & HIST & BARY & CPU \\
\hline 5 & $10 \mathrm{~min}$ & 4 & $49 \mathrm{MB}$ & $1.5 \mathrm{MB}$ & $25 \mathrm{~min}$ \\
10 & $10 \mathrm{~min}$ & 4 & $127 \mathrm{MB}$ & $5.6 \mathrm{MB}$ & $1.9 \mathrm{~h}$ \\
15 & $10 \mathrm{~min}$ & 4 & $256 \mathrm{MB}$ & $13 \mathrm{MB}$ & $7.9 \mathrm{~h}$ \\
\hline 10 & $5 \mathrm{~h}$ & 4 & $7 \mathrm{MB}$ & $5.6 \mathrm{MB}$ & $9 \mathrm{~min}$ \\
10 & $1 \mathrm{~h}$ & 4 & $30 \mathrm{MB}$ & $5.6 \mathrm{MB}$ & $30 \mathrm{~min}$ \\
10 & $10 \mathrm{~min}$ & 4 & $127 \mathrm{MB}$ & $5.6 \mathrm{MB}$ & $1.9 \mathrm{~h}$ \\
10 & $1 \mathrm{~min}$ & 4 & $661 \mathrm{MB}$ & $5.6 \mathrm{MB}$ & $7.5 \mathrm{~h}$ \\
\hline 10 & $10 \mathrm{~min}$ & 2 & $127 \mathrm{MB}$ & $2.8 \mathrm{MB}$ & $55 \mathrm{~min}$ \\
10 & $10 \mathrm{~min}$ & 3 & $127 \mathrm{MB}$ & $4.2 \mathrm{MB}$ & $1.4 \mathrm{~h}$ \\
10 & $10 \mathrm{~min}$ & 4 & $127 \mathrm{MB}$ & $5.6 \mathrm{MB}$ & $1.9 \mathrm{~h}$ \\
10 & $10 \mathrm{~min}$ & 10 & $127 \mathrm{MB}$ & $14 \mathrm{MB}$ & $4.8 \mathrm{~h}$
\end{tabular}

TABLE I: Influence of parameters on CPU time and data size (HISTograms before clustering and BARYcenters after). Dataset is $10 \%$ of the total 18322 segments of the Luxembourg scenario, with raw size about $480 \mathrm{MB}$.

std error regardless of the parameters choice. Therefore, in the following sections we will focus on the Memoryless method.

For the 1-day simulation on the whole city of Luxembourg, the raw data corresponding to the 18322 segments take up to $5.7 \mathrm{~GB}$. Table $\mathrm{I}$ indicates the data size before ('HIST') and after ('BARY') clustering, as well as the CPU time for the clustering, for a $10 \%$ subset of the 18322 segments. For the whole dataset, with a discretization $N_{V}=N_{A}=10$ and a time-frame $\Delta t=10 \mathrm{~min}$ the histograms for the (speed,acceleration) distributions are computed in less than $15 \mathrm{~min}$ and amount to $1.2 \mathrm{~GB}$. Setting $k=4$, the clustering step takes $19 \mathrm{~h}$ and the distributions for the barycenters occupy 59MB. Taking the histograms already reduces data occupancy by $79 \%$, and clustering pushes it to $99 \%$ in total. More generally, the data reduction done by the histograms is related to the speed/acceleration discretization, while the clustering further reduces data size according to the ratio $k / \Delta t$. Memory usage during the computations was about $100 \mathrm{MB}$ and posed no difficulties. It should be noted that the clustering is an offline step that only has to be done once. Also, it is independent for all segments and may benefit heavily from parallelization.

In the end, we observe that the range of parameters that give a reasonable trade-off between accuracy, data size and computation times is rather small, which limits the interest of trying sophisticated techniques to deduce some 'optimal' settings. The number of clusters $k$ is discussed again in $\mathrm{V}$ As for the $N_{V}, N_{A}$ discretization, possible improvements could be for instance the kind of adaptive exploration described in [24], provided the overall cost of the exploration does not outweight the cost of simply taking a sufficient fixed discretization.

\section{RESUlts USING REAL DATA}

Thanks to floating car data collected by the smartphone application Geco air [25], we were able to test our method on a real-life scenario. We focused on a portion of the A7 highway near Lyon, France, which is known to be regularly used by commuters. For our analysis, the traffic data collected during the working days of the last two years were aggregated as they 
were recorded over one day, reasonably assuming that the data share similar traffic patterns. The speed measurements were then divided into 10-minutes time-frames. The Memoryless method is applied to the 1632 collected speed profiles. The discretization of the (speed,acceleration) space is limited at a $10 \times 10$ grid to reduce the computation time. One of the main goal of our method is to represent traffic with a small number of (speed,acceleration) distributions, thus we choose to explore the traffic representation with a number of clusters between 1 and 10. As we did with the data from SUMO, we will compare the consumption distribution of the Memoryless method $C_{M S M}$ to the reference consumptions $C_{R e f}$.

\section{A. Clustering impact}

The choice of the number of clusters $k$ is related to a tradeoff between data size, computational time and accuracy of the consumption estimate. A small number of clusters will reduce the data occupancy and CPU time, with the risk of a coarser consumption estimate. A larger $k$ will give consumptions closer to the unclustered raw data, at the cost of increased data size and CPU time. Fig. 8 shows the mean and standard deviation errors between the consumption distributions $C_{R e f}$ and $C_{M S M}$, for a number of clusters $k \in 1, \cdots, 10$, as well as the unclustered case (dashed line). We see that the mean error is below 5\%, and further decreases below $2 \%$ for $k=2,3,4$ clusters, while larger $k$ have larger errors. Concerning the standard deviation, it is below 0.4 and appears globally decreasing with $k$. The few quirks in the curve may be due to some lingering sensitivity of the barycenter computation to the initialization. In the following, we set $k=4$ clusters since this value gives a good trade-off. The discussion in $\mathrm{V}-\mathrm{C}$ shows the link between the clusters and the traffic conditions, and sheds some light on why a small number of clusters seems optimal, since typical traffic conditions likely encompass only a limited number of situations (fluid, jammed, etc).

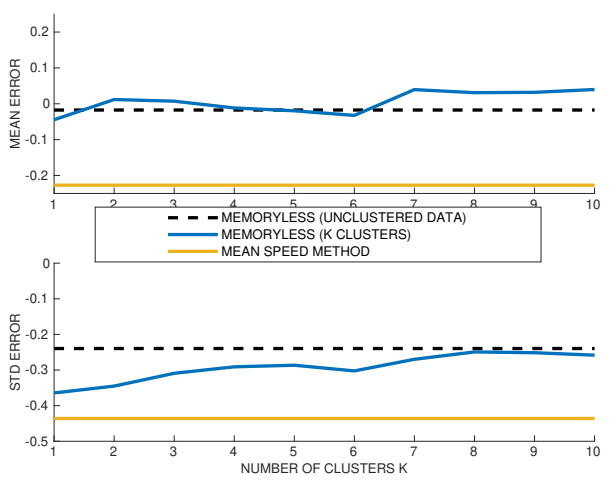

Fig. 8: Memoryless sampling method. Relative mean error and standard deviation for different numbers of clusters $k$. Errors for the unclustered data and the mean speed method are indicated as well. The value $k=4$ seems to be a good choice in terms of errors and computational cost.

\section{B. Mean speed method}

Furthermore, we decide to compare our statistical model based on (speed,acceleration) distributions, to a simpler approach using only mean speeds, available from tools such as HERE or Google Maps. Noting $\bar{v}_{s, t}$ the mean speed for a given pair (segment,timeframe), we compute a basic estimate of the energy consumption $C_{A V G S}$ with a constant speed and null acceleration

$$
C_{A V G S}=\frac{P\left(\bar{v}_{s, t}, 0\right) L}{\bar{v}_{s, t}} .
$$

Taking our set of (speed,acceleration) profiles from section $\mathrm{V}$, we recompute the mean speed information. We show on Fig. 9 the probability distributions for the reference consumption, memoryless method and mean speed method. We observe that the memoryless results are closer to the reference, with relative errors $\left(\varepsilon_{\text {mean }}=4.6 \%, \varepsilon_{\sigma}=24.4 \%\right)$ better than $\left(\varepsilon_{\text {mean }}=22.7 \%, \varepsilon_{\sigma}=43.6 \%\right)$ for the mean speed method.

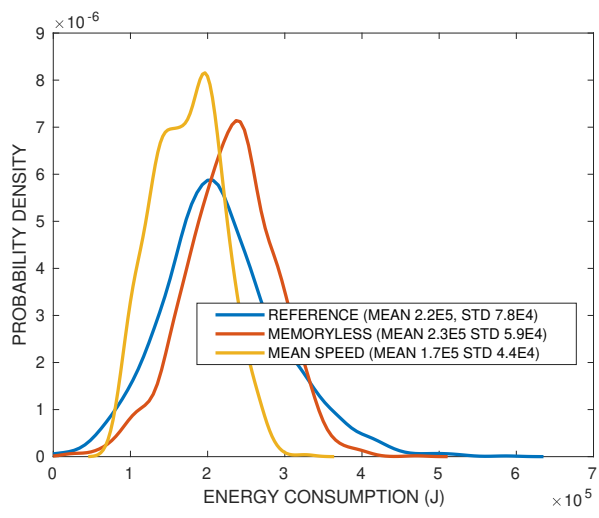

Fig. 9: Probability distributions of energy consumption. Reference (blue) vs Memoryless sampling method (red) and mean speed method (yellow). The mean and std are indicated for the 3 discrete distributions.

\section{Analysis of the 4-cluster case}

In the following we focus on the 4-cluster case that seems to offer a good trade-off between model size and accuracy. On Fig. 10 we plot the subset of speed profiles belonging to each of the 4 clusters. We observe that these speed profiles do appear rather similar in each cluster. Clusters 1 to 3 correspond to relatively smooth traffic conditions, with little speed variations, 2 being the fastest, followed by 1 then 3 . Cluster 4, on the other hand, obviously corresponds to a traffic jam situation, with large variations in speed and frequent drops to null speeds.

Fig. 11 represents the same 4 subsets of profiles for each cluster in the (speed,acceleration) space. This representation confirms that the (speed,acceleration) distributions are quite distinct for each cluster. The level sets on each graph correspond to the distribution of the barycenter of the cluster, and we observe that the barycenters coincide rather well with the profile subsets. Furthermore, we can interpret the clusters in terms of traffic conditions, as summarized on Tab. II 

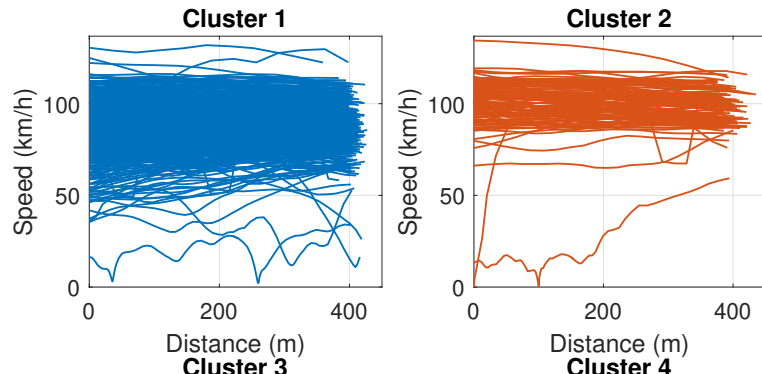

Cluster 3
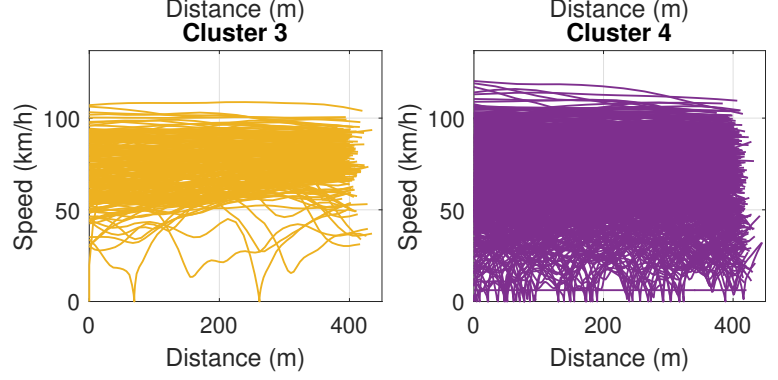

Fig. 10: Speed profiles associated with the clusters.
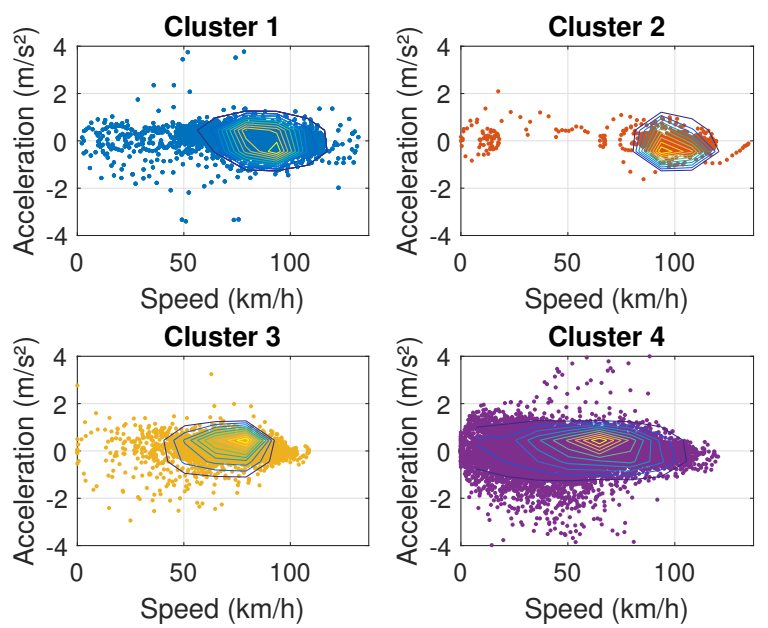

Fig. 11: Real distributions compared to their cluster.

TABLE II: Traffic Interpretation

\begin{tabular}{c|c|c|c|c} 
Cluster & Mean Speed & Speed Spread & Acceleration & Interpretation \\
\hline 1 & Medium & Important & Small & Normal \\
2 & High & Low & Small & Fluid \\
3 & Low & Low & Small & Dense \\
4 & Very low & High & Well spread & Traffic jam
\end{tabular}

In order to see whether the traffic interpretation is coherent with reality, the clusters associated with each time-frame were plotted in Fig. 12, in which the grey portions correspond to the time-frames with not enough data for the analysis. We can observe that the cluster 3, corresponding to a dense traffic, appears in the morning between 7am and 9:30am. The cluster 4 , traffic jam, is present essentially between $4: 30 \mathrm{pm}$ and $8 \mathrm{pm}$. Cluster 1, normal traffic conditions, during the day and cluster 2 , fluid conditions, during the night. Overall, this traffic pattern seems very consistent with reality, exhibiting two peak hours in the morning and evening, typical of commuting behavior.

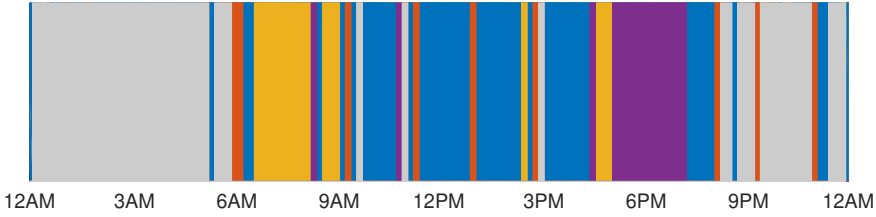

Fig. 12: Traffic clusters according to the time of day.

\section{Summary}

The analysis conducted on real data confirms that representing traffic by means of (speed,acceleration) distributions is effective not only to estimate the energy consumption distributions in different portions of the road network but also to identify different traffic conditions. Also, clustering proves to be an effective method to reduce data occupancy.

The cluster stage fits its role, allowing us to keep a reasonable data size while retaining most of the useful information from the original set of $(v, a)$ profiles.

\section{CONCLUSiOnS}

In this paper, we have presented a new approach to use traffic data to predict the energy consumption of vehicles. The key point is to consider the (speed,acceleration) data in a statistical sense without the temporal aspect, coupled with a decomposition of the road network into a collection of small segments, based on topological aspects.

Numerical experiments carried out with traffic data generated by the traffic simulator SUMO indicate that our approach is able to reconstruct the distribution of the energy consumption over a set of vehicles. We introduce two methods to compute the energy consumption, called Average and Memoryless Sampling methods. The Memoryless Sampling method gives a more accurate estimate of the distribution of energy consumptions, according to indicators such as std error and Jensen-Shannon divergence.

We also investigate the influence of several parameters such as the (speed,acceleration) discretization, length of time-frame for data aggregation, and number of clusters for the data reduction.

The analysis on real data shows that the Memoryless Sampling method performs better than a more basic approach based on mean speed only. Another interesting point is that the clusters are consistent with the traffic conditions.

Possible future works include a second level of clustering, creating clusters of road segments with close traffic conditions, and variants of the Memoryless Sampling method that would retain some temporality (e.g. Markov). Another direction could be to investigate the possible coupling of the presented model with approaches in flow management such as the SS-CTM in [26], since both use a spatial decomposition and account for stochastic aspects of the traffic.

\section{REFERENCES}

[1] "European Strategies - White Paper 2011." [Online]. Available: https://goo.gl/GoSWcf 
[2] L. Evans, R. Herman, and T. Lam, "Multivariate Analysis of Traffic Factors Related to Fuel Consumption in Urban Driving," Transportation Science, vol. 10, no. 2, pp. 205-215, 1976. [Online]. Available: https://doi.org/10.1287/trsc.10.2.205

[3] I. D. Vlieger, D. D. Keukeleere, and J. Kretzschmar, "Environmental effects of driving behaviour and congestion related to passenger cars," Atmospheric Environment, vol. 34, no. 27, pp. 4649 - 4655, 2000. [Online]. Available: http://www.sciencedirect.com/science/article/ pii/S135223100000217X

[4] M. Treiber and A. Kesting, "Traffic flow dynamics," Traffic Flow Dynamics: Data, Models and Simulation, Springer-Verlag Berlin Heidelberg, 2013.

[5] M. J. Lighthill and G. B. Whitham, "On kinematic waves. II. A theory of traffic flow on long crowded roads," Proceedings of the Royal Society of London. Series A, Mathematical and Physical Sciences, pp. 317-345, 1955.

[6] S. Panwai and H. Dia, "Comparative evaluation of microscopic carfollowing behavior," IEEE Transactions on Intelligent Transportation Systems, vol. 6, no. 3, pp. 314-325, Sept 2005

[7] S. K. Zegeye, B. De Schutter, J. Hellendoorn, E. A. Breunesse, and A. Hegyi, "Integrated Macroscopic Traffic Flow, Emission, and Fuel Consumption Model for Control Purposes," Transportation Research Part C, vol. 31, pp. 158-171, 2013.

[8] C. Guo, B. Yang, O. Andersen, C. S. Jensen, and K. Torp, "EcoMark 2.0: Empowering Eco-Routing with Vehicular Environmental Models and Actual Vehicle Fuel Consumption Data," Geoinformatica, vol. 19, pp. 567-599, 2015.

[9] E. Ericsson, "Variability in urban driving patterns," Transportation Research Part D: Transport and Environment, vol. 5, no. 5, pp. 337 354, 2000. [Online]. Available: http://www.sciencedirect.com/science/ article/pii/S1361920900000031

[10] L. N. Vasershtein, "Markov processes over denumerable products of spaces describing large system of automata," Problemy Peredaci Informacii, vol. 5, pp. 64-72, 1969.

[11] R. L. Dobrushin, "Prescribing a System of Random Variables by Conditional Distributions," Theory of Probability \& Its Applications, vol. 15 , no. 3, pp. 458-486, 1970.

[12] M. Agueh and G. Carlier, "Barycenters in the Wasserstein space," SIAM Journal on Mathematical Analysis, vol. 43, no. 2, pp. 904-924, 2011.

[13] J.-D. Benamou, G. Carlier, M. Cuturi, L. Nenna, and G. Peyré, "Iterative Bregman projections for regularized transportation problems," SIAM J. Sci. Comput., vol. 37, no. 2, pp. A1111-A1138, 2015. [Online]. Available: http://dx.doi.org/10.1137/141000439

[14] J. MacQueen, "Some methods for classification and analysis of multivariate observations," in Proc. Fifth Berkeley Sympos. Math. Statist. and Probability (Berkeley, Calif., 1965/66). Univ. California Press, Berkeley, Calif., 1967, pp. Vol. I: Statistics, pp. 281-297.

[15] D. Lefebvre and M. David, "Dynamic clustering and strong patterns recognition: new tools in automatic classification," Canadian Journal of Earth Sciences, vol. 14, no. 10, pp. 2232-2245, 1977.

[16] G. De Nunzio and L. Thibault, "Energy-optimal driving range prediction for electric vehicles," in Intelligent Vehicles Symposium (IV), 2017 IEEE. IEEE, 2017, pp. 1608-1613.

[17] J. G. Hayes and K. Davis, "Simplified electric vehicle powertrain model for range and energy consumption based on epa coast-down parameters and test validation by argonne national lab data on the nissan leaf," in 2014 IEEE Transportation Electrification Conference and Expo (ITEC), June 2014, pp. 1-6.

[18] S. Kullback and R. A. Leibler, "On information and sufficiency," Ann. Math. Statistics, vol. 22, pp. 79-86, 1951.

[19] I. Dagan, L. Lee, and F. Pereira, "Similarity-based Methods for Word Sense Disambiguation," in Proceedings of the 35th Annual Meeting of the Association for Computational Linguistics and Eighth Conference of the European Chapter of the Association for Computational Linguistics, ser. ACL '98. Stroudsburg, PA, USA: Association for Computational Linguistics, 1997, pp. 56-63. [Online]. Available: https://doi.org/10.3115/976909.979625

[20] D. M. Endres and J. E. Schindelin, "A new metric for probability distributions," IEEE Transactions on Information Theory, vol. 49, no. 7, pp. $1858-1860,2003$.

[21] F. Österreicher and I. Vajda, "A new class of metric divergences on probability spaces and its applicability in statistics," Annals of the Institute of Statistical Mathematics, vol. 55, no. 3, pp. 639-653, Sep 2003.

[22] D. Krajzewicz, J. Erdmann, M. Behrisch, and L. Bieker, "Recent Development and Applications of SUMO - Simulation of Urban MObility,"
International Journal On Advances in Systems and Measurements, vol. 5, no. 3\&4, pp. 128-138, December 2012.

[23] L. Codecá, R. Frank, S. Faye, and T. Engel, "Luxembourg SUMO Traffic (LuST) Scenario: Traffic Demand Evaluation,” IEEE Intelligent Transportation Systems Magazine, vol. 9, no. 2, pp. 52-63, 2017.

[24] B. Yang, J. Dai, C. Guo, C. S. Jensen, and J. Hu, "Pace: a path-centric paradigm for stochastic path finding," The VLDB Journal, vol. 27, no. 2, pp. 153-178, Apr 2018.

[25] "Geco air." [Online]. Available: http://www.gecoair.fr/home en/

[26] L. Jin, A. A. Kurzhanskiy, and S. Amin, "Throughput-improving control of highways facing stochastic perturbations," CoRR, vol. abs/1809.07610, 2018. [Online]. Available: http://arxiv.org/abs/1809. 07610

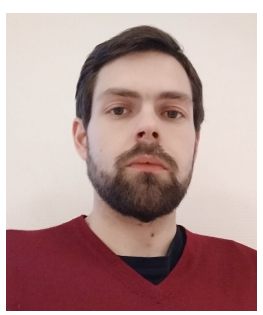

Arthur Le Rhun received a M.Sc. degree in engineering from the ENSTA Paristech (France), a M.Sc. degree in Optimization from Université Paris XI both in 2016. He is currently pursuing a Ph.D. in applied mathematics at the Ecole polytechnique and IFP Energies nouvelles. His research theme is dynamic optimization for stochastic systems, and applications to energy management of hybrid vehicles.

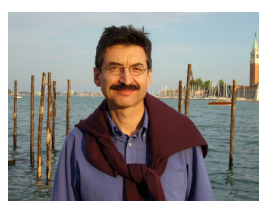

Frederic Bonnans Got an engineering degree from the Ecole Centrale Paris, a PhD (docteur ingenieur)from the Université Technique de Compiègne, and the Habilitation from Université Paris IX Dauphine in 1979, 1982 and 1993 resp. He is currently a senior researcher at Inria Saclay and Ecole polytechnique. His research theme is dynamic optimization for deterministic and stochastic systems, and applications to energy management and biological models.

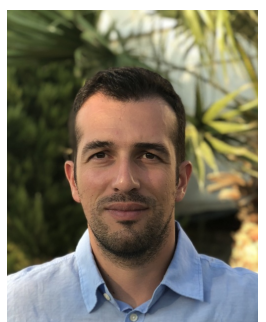

Giovanni De Nunzio received the B.Sc. and M.Sc. degrees in information and automation engineering from the University of L'Aquila, Italy, in 2007 and 2010. He received the PhD in automatic control from the Grenoble Institute of Technology, France, in 2015. He is currently a research engineer at IFP Energies nouvelles, Lyon, France. His research activities focus on modeling, control, and simulation of traffic and transportation systems, vehicle powertrain modeling, advanced driver-assistance systems, energy consumption minimization, and graph theory.

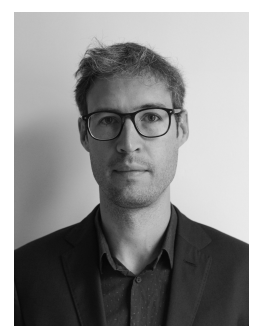

Thomas Leroy graduated from ESSTIN and received the M.Sc. degree in control theory from the University Henri Poincare in Nancy, France, in 2006. He received the doctorate degree in Control theory and Mathematics from the Ecole des Mines ParisTech, France, in 2010. He is currently a research engineer at IFP Energies nouvelles, RueilMalamaison, France. His research activities focus on modeling, control, and simulation of internal combustion engines and hybrid electric powertrains.

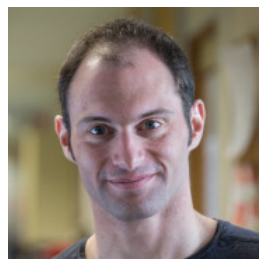

Pierre Martinon received his M.Sc. and Ph.D. degrees in applied mathematics from the ENSEEIHT (Toulouse, France) in 2001 and 2005. He is currently a researcher at Inria Saclay and Ecole polytechnique. His research field is optimization for dynamical systems, more specifically the numerical aspects. 